\title{
Cerebral venous thrombosis: an unexpected complication from spinal surgery
}

\author{
Bruno Lourenço Costa $\cdot$ Motasem Shamasna • \\ Joana Nunes · Florbela Magalhães • \\ António Júdice Peliz
}

Received: 6 June 2013/Revised: 15 December 2013/Accepted: 19 December 2013

(c) Springer-Verlag Berlin Heidelberg 2013

\begin{abstract}
Purpose To provide new insights into the pathophysiology, prevention and diagnosis of cerebral venous thrombosis (CVT) associated with iatrogenic cerebrospinal fluid (CSF) leaks and/or external CSF drainage.

Methods Case report and literature review.

Results We describe the case of a 30-year-old woman who developed a CSF fistula after lumbar spinal surgery. The treatment included rest, hydration, caffeine, and continuous lumbar CSF drainage. After closure of the fistula, the patient complained of severe orthostatic headache. Thrombosis involving the superior sagittal sinus, the right transverse sinus, the right sigmoid sinus, and the right jugular vein was diagnosed after neurological deterioration.
\end{abstract}

B. Lourenço Costa $(\square) \cdot$ A. J. Peliz

Department of Neurosurgery, Centro Hospitalar e Universitário de Coimbra, Praceta Mota Pinto, 3000-075 Coimbra, Portugal e-mail: brunolourencocosta@gmail.com

A. J. Peliz

e-mail: antoniopeliz@sapo.pt

M. Shamasna · F. Magalhães

Department of Neurology, Centro Hospitalar e Universitário de Coimbra, Quinta Dos Vales, São Martinho Do Bispo,

3041-801 Coimbra, Portugal

e-mail: smotasem@gmail.com

F. Magalhães

e-mail: florbelamagalhaes@sapo.pt

J. Nunes

Department of Neuroradiology, Centro Hospitalar e

Universitário de Coimbra, Quinta Dos Vales, São Martinho Do

Bispo, 3041-801 Coimbra, Portugal

e-mail: joanitabgc@hotmail.com
Conclusion A few reports have associated CVT with various forms of spinal meningeal injury. However, it has been rarely documented following spinal surgery complicated by accidental durotomy and/or external lumbar CSF drainage. CSF hypovolemia may precipitate CVT in patients having prothrombotic risk factors. Patients who have or had CSF leaks and/or lumbar CSF drains who present with symptoms of intracranial CSF hypotension should remain in the horizontal position to prevent CVT. In that context, the diagnosis of CVT depends on a high degree of suspicion.

Keywords Cerebrospinal fluid leak - Cerebral venous thrombosis - External lumbar cerebrospinal fluid drainage . Intracranial hypotension · Spinal surgery

\section{Introduction}

Several prothrombotic risk factors for cerebral venous thrombosis (CVT) are consistently described in the literature. In the majority of patients, CVT has a multifactorial etiology [1-3]. The exact mechanisms by which each risk factor causes or precipitates thrombosis are complex and only partially understood. The role of cerebrospinal fluid (CSF) hypovolemia as a risk factor for CVT has deserved little attention. We describe here the rare case of a patient who developed dural sinuses thrombosis following a spinal lumbar surgery complicated by a CSF fistula which was treated with external CSF drainage. We take this opportunity to timely review the literature and provide new insights into the pathophysiology, prevention, and diagnosis of CVT associated with iatrogenic CSF leaks and/or external CSF drainage. 


\section{Case description}

A previously healthy 30-year-old woman complained of chronic and disabling lumbago. Lumbar spine CT and MRI revealed L5-S1 discarthrosis, grade I/IV listhesis associated with bilateral isthmic lysis, and bifid L5 posterior arch as well. During the open approach for transpedicular screw L4-L5-S1 fixation, an accidental durotomy at the L5 level occurred. The dural injury was repaired with sutures and fibrin glue, but the hardware placement was abandoned. During the following week, continuous CSF drainage through the operative wound was observed, in spite of suture revision and rest. The closure of the fistula required bed rest and continuous external CSF drainage for 2 weeks. A 16-gauge catheter was inserted at the L3-L4 space after three attempts and the height of the collecting chamber of the system was adjusted to promote drainage of about $10 \mathrm{~mL} / \mathrm{h}$. Prophylactic ceftriaxone and enoxaparin were prescribed during this period. After removal of the lumbar drain, the patient complained of severe orthostatic headache and dizziness. The complaints diminished after introduction of intravenous fluids and caffeine. However, 3 days after removal of the lumbar drain, she complained of severe headache, nausea and photophobia, which were not alleviated in the recumbent position, as well as right hemihypoesthesia/paresthesia. Signs of surgical wound dehiscence/infection, and CSF leakage or subcutaneous/ paravertebral fluid collection were not noted. She did not have fever or meningeal signs but was prostrated, and fundoscopy revealed bilateral papilledema, retinal vessel tortuosity and retinal hemorrhages. A focal epileptic crisis of motor aphasia was observed. Contrast-enhanced CT scan revealed thrombosis involving the superior sagittal sinus, the right transverse sinus, the right sigmoid sinus, and the right jugular vein, as well as slight diffuse cerebral edema (Fig. 1). Anticoagulation with enoxaparin and warfarin (target INR 2-3) was introduced. After 7 days of rest and treatment with acetaminophen and prophylactic levetiracetam, the patient was discharged. She showed no relevant symptoms or neurological deficits. A detailed investigation identified the following prothrombotic factors: prothrombin 20210G/A heterozygosity, methylenetetrahydrofolate reductase (MTHFR) homozygosity plus hyperhomocysteinemia, and oral contraception. Her personal and family history was negative for venous thromboembolic or cerebrovascular events. Oral contraception was stopped and anticoagulation with warfarin was continued ad eternum. One year after the surgery, the patient complained of residual lumbago, and her general and neurological examinations were found normal. Additional thromboembolic events were not noted. The repermeabilization of the thrombosed sinuses and the absence of cerebral lesions were documented by MRI 6 months after the diagnosis of CVT (Fig. 2).

\section{Discussion}

Accidental durotomy is a frequent complication of spinal surgery, being easily treated with minimum morbidity in the majority of cases. External lumbar CSF drainage systems are widely and safely employed in clinical neurology and neurosurgery. However, to our knowledge, only Miglis and Levine have previously reported a case of CVT following spinal surgery complicated by durotomy, which was treated with the aid of external CSF drainage, presenting a more dramatic outcome and a much shorter follow-up period [4]. Nevertheless, a few reports have associated CVT with other forms of spinal meningeal injury, including spontaneous intracranial hypotension, diagnostic lumbar puncture, myelography, and epidural and spinal anesthesia, as well as intrathecal administration of steroids and cytostatics [4-23]. The temporal gap between the iatrogenic injury to the spinal meninges and the diagnosis of CVT has ranged from a few hours to a few days.

Actually, CSF hypovolemia due to spinal meningeal injury of any type may precipitate CVT, especially when patients with prothrombotic conditions assume an erect position. Despite the absence of a uniform investigation, the majority of patients had genetic or acquired prothrombotic risk factors, but their history was negative for previous cerebrovascular events. However, in almost all cases, dural injury produced symptomatic intracranial CSF hypotension. When patients are recumbent, CSF hypovolemia caused by meningeal injuries is homogeneously distributed throughout the CSF space. However, when patients assume an erect position, CSF hypovolemia in the intracranial compartment is abruptly exacerbated due to gravity-dependent CSF caudal displacement, producing two relevant consequences. First, caudal displacement of the brain with acute stretching and distortion of the meninges, vessels and nerves occurs [24]. Second, according to the Monro-Kellie hypothesis, a compensatory increase in total blood volume in the intracranial compartment happens to maintain the volume of its content. An important portion of the additional blood volume is accommodated in the capacitance veins and, to a lesser degree, in the venous sinuses, producing an increase in their diameter and a proportional decrease in the blood flow velocity in their interior, as predicted by the hydrodynamic theory and confirmed by MRI and transcranial Doppler ultrasound studies [24-26]. During the shift to the erect position, these changes trigger the stimulation of pain receptors located in the meninges, vessels and nerves, which is thought to cause the clinical manifestations of intracranial 

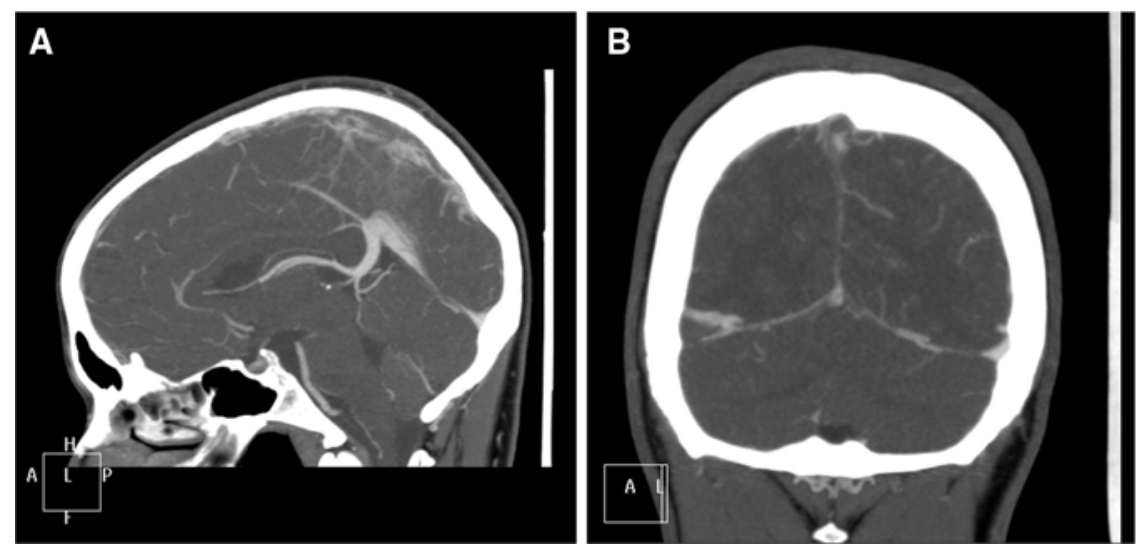

Fig. 1 Sagittal (a), and coronal (b, c) contrast-enhanced CT scan (venous phase). Abnormal diffuse dural enhancement and engorgement of the dural sinuses and veins are frequent findings in patients with intracranial CSF hypotension $(\mathbf{a}-\mathbf{c})$. The superior sagittal sinus $(\mathbf{a}-\mathbf{c})$, the right transverse sinus and right sigmoid sinus (b) show

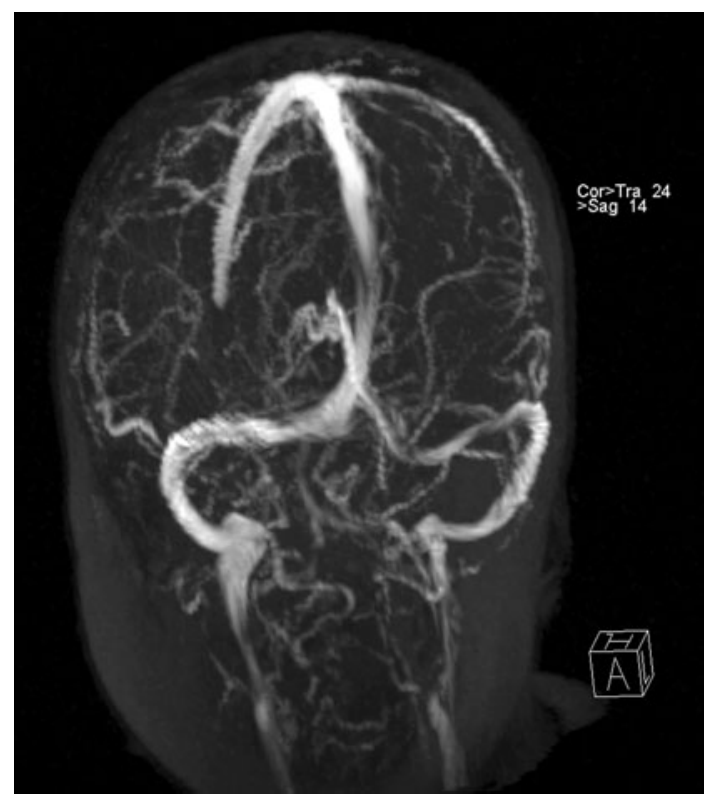

Fig. 2 Postprocessed image from a two-dimensional time-of-flight magnetic resonance venogram (TOF-MRV) performed 6 months after the diagnosis of CVT showing complete repermeabilization of the right transverse sinus, right sigmoid sinus, and right jugular vein

CSF hypotension, including the classic post-lumbar puncture headache $[27,28]$. However, the same mechanisms may promote CVT, according to Virchow's theory. First, distortion of sinusal and venous walls impairs endothelial antithrombotic properties. Second, blood flow in the dural sinuses and capacitance veins is slow under physiologic conditions and may be critically reduced due to acute engorgement of these vessels. Third, the viscosity of venous blood increases due to the reduction of CSF reabsorption. peripheral enhancement along with central nonopacification ("empty delta sign"), suggesting thrombosis that also involves the right jugular vein (c). The left transverse sinus and the left sigmoid sinus are hypoplastic. Slight bilateral and diffuse cerebral edema was also evident (not shown)

After surgical injury to the spinal meninges, the treatment of the CSF leak and the restitution of CSF volume are the best strategies to improve the symptoms of intracranial hypotension and prevent CVT. When external CSF drainage systems are employed, overdrainage should be carefully avoided. Patients who have or have had CSF leaks and/or lumbar drains and present with intracranial hypotension symptoms should not have their head elevated, remaining in the horizontal or Trendelenburg position. After closure of the leak and/or removal of the drain, the shift to the erect position should be progressive.

We must emphasize that the diagnosis of CVT after spinal surgery and/or external CSF drainage is not always immediate. First, dural injuries are not always recognized during surgery. However, spinal surgery and/or lumbar CSF drain insertion may cause meningeal injuries that, despite producing important epidural or paravertebral CSF collections, manifest exclusively by intracranial CSF hypotension symptoms. Second, the clinical presentation of CVT is nonspecific and isolated intracranial hypertension may be the sole manifestation of CVT, especially in patients with dural sinus thrombosis without involvement of superficial or deep veins [1-3]. CVT should be excluded every time in patients with symptoms of intracranial CSF hypotension after spinal surgery and/or external lumbar CSF drainage show the following: (1) complaint of headache that worsens after an initial plateau, loses its orthostatic character or lasts for more than a few days after the introduction of proper treatment; and (2) a decreased level of consciousness, encephalic focal neurologic deficits or seizures $[4,5,20$, 23]. However, the change in headache pattern may not reliably predict the development of CVT [20].

In the reported case, the patient had genetic and acquired prothrombotic factors. CSF hypovolemia was caused by 
continuous CSF leakage through the fistula and the external CSF drainage system. Additional dural injury could have been produced during puncture attempts. The shift to the erect position exacerbated CSF hypovolemia in the intracranial compartment, causing symptoms of intracranial hypotension and precipitating CVT. Dural sinus thrombosis manifested as nonspecific symptoms of intracranial hypertension and a seizure. It is important to note that CVT was diagnosed after closure of the fistula and removal of the drain, probably due to residual occult CSF leakage to epidural or paravertebral spaces and/or incomplete CSF volume replacement. Young age, absence of infectious or neoplastic underlying disease, good neurological status, thrombosis limited to venous sinuses, and absence of ischemic or hemorrhagic lesions, as well as a prompt diagnosis and a proper treatment explained the observed outcome [3]. However, according to a recent prospective study, CVT still carries a mortality of $8.3 \%$ and a permanent neurological morbidity of $5.1 \%$ [3].

\section{Conclusion}

Our report adds to the limited literature on the role of CSF hypovolemia as a risk factor for CVT and supports the notion that this cerebrovascular condition is a rare but difficult to diagnose and potentially severe complication of spinal surgery and external CSF drainage.

Conflict of interest The authors have no actual or potential conflict of interest.

\section{References}

1. Bousser MG, Ferro JM (2007) Cerebral venous thrombosis. Lancet Neurol 6:162-170

2. Ferro JM, Canhão $P$, Stam J, Bousser MG, Barinagarrementeria $F$ (2004) Prognosis of cerebral vein and dural sinus thrombosis: results of the international study on cerebral vein and dural sinus thrombosis (ISCVT). Stroke 35:664-670

3. Stam J (2005) Thrombosis of the cerebral veins and sinuses. NEJM 362:1791-1798

4. Miglis MG, Levine DB (2010) Intracranial venous thrombosis after placement of a lumbar drain. Neurocrit Care 12:83-87

5. Aidi S, Chaunu M, Biousse V, Bousser M (1999) Changing pattern of headache pointing to cerebral venous thrombosis after lumbar puncture and intravenous high-dose steroids. Headache 39:559-564

6. Albucher JF, Vuillemin-Azais C, Manelfe C, Clanet M, GuiraudChaumeil B, Chollet F (1999) Cerebral thrombophlebitis in three patients with probable multiple sclerosis. Cerebrovasc Dis 9:298-303

7. Benzon HT, Iqbal M, Tallman MS, Boehlke L, Russell EJ (2003) Superior sagittal sinus thrombosis in a patient with postdural puncture headache. Reg Anesth Pain Med 28:64-67

8. Bienfait HP, Gijtenbeck JMM, van den Bent MJ, de Bruin HG, Voogt PJ, Pillay M (2002) Cerebral venous and sinus thrombosis with cerebrospinal fluid circulation block after the first methotrexate administration by lumbar puncture. Neuroradiology 44:929-932

9. Borum SE, Naul LG, McLeskey CH (1997) Postpartum dural venous sinus thrombosis after postdural puncture headache and epidural blood patch. Anesthesiology 86:487-490

10. Brugeilles H, Penisson-Besnier I, Pasco A, Oillic P, Lejeune P, Mercier P (1996) Cerebral venous thrombosis after myelography with iopamidol. Neuroradiology 38:534-536

11. Gewirtz EC, Costin M, Marx GF (1987) Cortical vein thrombosis may mimic postdural puncture headache. Reg Anesth 12:188-190

12. Ghatge S, Uppugonduri S, Kamarzaman Z (2008) Cerebral venous thrombosis following accidental dural puncture and epidural blood patch. Interv J Obstet Anesth 17:267-270

13. Gunal DI, Afsar N, Tuncer N, Aktan S (2002) A case of multiple sclerosis with cerebral venous thrombosis: the role of lumbar puncture and high-dose steroids. Eur Neurol 47:57-58

14. Hartley RW, Jackson A, Cooke RS (1992) Dural venous sinus thrombosis following myelography: demonstration by magnetic resonance imaging. Br J Radiol 65:1134-1136

15. Hubbert CH (1987) Dural puncture headache suspected, cortical vein thrombosis diagnosed. Anesth Analg 66:283-286

16. Maurelli M, Bergamaschi R, Candeloro E, Todeschini A, Micieli G (2005) Cerebral venous thrombosis and demyelinating diseases: report of a case in a clinically isolated syndrome suggestive of multiple sclerosis onset and review of the literature. Mult Scler 11:242-244

17. Milhaud D, Heroum C, Charif M, Saulnier P, Pages M, Blard JM (2000) Dural puncture and corticotherapy as risks factors for cerebral venous sinus thrombosis. Eur J Neurol 7:123-124

18. Mouraux A, Gille M, Dorban S, Peetters A (2002) Cortical venous thrombosis after lumbar puncture. J Neurol 249:1313-1315

19. Ravindran RS, Zandstra GC (1989) Postpartum headache following regional anesthesia; a symptom of cerebral venous thrombosis. Can J Anaesth 36:705-707

20. Schievink WI, Maya MM (2008) Cerebral venous thrombosis in spontaneous intracranial hypotension. Headache 48(10): $1511-1519$

21. Schou J, Scherb M (1986) Postoperative sagittal sinus thrombosis after spinal anesthesia. Anesth Analg 65:541-542

22. Sommerstein R, Jung HH, Knoblauch C (2011) Cerebral venous sinus thrombosis as a complication of a microendoscopic discectomy. Eur Neurol 65(2):72-73

23. Wilder-Smith E, Kothbauer-Margreiter I, Lammle B, Sturzenegger M, Ozdoba C, Hauser SP (1997) Dural puncture and activated protein $\mathrm{C}$ resistance: risk factors for cerebral venous sinus thrombosis. J Neurol Neurosurg Psychiatry 63:351-356

24. Forghani R, Farb RI (2008) Diagnosis and temporal evolution of signs of intracranial hypotension on MRI of the brain. Neuroradiology 50:1025-1034

25. Canhão P, Batista P, Falcão F (2005) Lumbar puncture and dural sinus thrombosis - a causal or casual association? Cerebrovasc Dis 19:53-56

26. Schoser BG, Riemenschneider N, Hansen HC (1991) The impact of raised intracranial pressure on cerebral venous hemodynamics: a prospective venous transcranial Doppler ultrasonography study. J Neurosurg 91(5):744-749

27. Levine D, Rapalino O (2001) The pathophysiology of lumbar puncture headache. J Neurol Sci 192:1-8

28. Schievink WI, Maya MM, Loouy C, Moser FG, Tourje J (2008) Diagnostic criteria for spontaneous spinal CSF leaks and intracranial hypotension. Am J Neuroradiol 29:853-856 